\title{
Interactions of diamond surfaces with fusion relevant plasmas
}

\author{
G De Temmerman ${ }^{1}$, R P Doerner ${ }^{2}$, P John $^{3}$, S Lisgo $^{1}$, A Litnovsky ${ }^{4}$, \\ L Marot $^{4}$, S Porro ${ }^{3}$, P Petersson $^{3}$, M Rubel $^{3}$, D L Rudakov ${ }^{2}$, \\ G Van Rooij ${ }^{8}$, J Westerhout ${ }^{8}$ and J I B Wilson ${ }^{3}$ \\ ${ }^{1}$ EURATOM/UKAEA Fusion Association, Culham Science Centre, Abingdon, UK \\ ${ }^{2}$ Center for Energy Research, University of California at San Diego, La Jolla, CA-92093, USA \\ ${ }^{3}$ Heriot-Watt University, Engineering and Physical Sciences, Riccarton, Edinburgh EH144AS, UK \\ ${ }^{4}$ Institut für Energieforschung, Forschungszentrum Jülich, Jülich, Germany \\ ${ }^{5}$ Department of Physics, University of Basel, Basel, Switzerland \\ ${ }^{6}$ Tandem Laboratory, Uppsala University, Association EURATOM - VR, Box 529, 75120 Uppsala, \\ Sweden \\ ${ }^{7}$ Alfven Laboratory, Royal Institute of Technology (KTH), Association EURATOM-VR, \\ 10044 Stockholm, Sweden \\ ${ }^{8}$ FOM Institute for Plasma Physics Rijnhuizen, Edisonbaan 14, 3439 MN Nieuwegein, The Netherlands \\ E-mail: gregory.detemmerman@ukaea.org.uk
}

Received 6 November 2009

Accepted for publication 17 November 2009

Published 30 December 2009

Online at stacks.iop.org/PhysScr/T138/014013

\begin{abstract}
The outstanding thermal properties of diamond and its low reactivity towards hydrogen may make it an attractive plasma-facing material for fusion and calls for a proper evaluation of its behaviour under exposure to fusion-relevant plasma conditions. Micro and nanocrystalline diamond layers, deposited on Mo and Si substrates by hot filament chemical vapour deposition (CVD), have been exposed both in tokamaks and in linear plasma devices to measure the erosion rate of diamond and study the modification of the surface properties induced by particle bombardment. Experiments in Pilot-PSI and PISCES-B have shown that the sputtering yield of diamond (both physical and chemical) was a factor of 2 lower than that of graphite. Exposure to detached plasma conditions in the DIII-D tokamak have evidenced a strong resistance of diamond against erosion under those conditions.
\end{abstract}

PACS numbers: 52.40.Hf, 52.77.Dq

(Some figures in this article are in colour only in the electronic version.)

\section{Introduction}

The choice of a plasma-facing material (PFM) for a fusion reactor is critical due to the very harsh conditions in which they would operate. In addition, the edge plasma and the first-wall material constitute a strongly coupled system. From the current knowledge, tungsten and tungstenbased alloys have been presented as the only recognized option [1], although different innovative solutions have been proposed [2]. Another route favours the use of liquid metals (Li, Ga) but although the experimental results to date are encouraging [3], the use of large surfaces of liquid metals as PFM is technologically challenging. Carbon-based materials, on the other hand, which have been used extensively in modern tokamaks (graphite, CFC) due to their good thermal properties and low atomic number, are not suitable for a reactor due to poor performance regarding erosion, retention and neutrons.

But unlike graphite/CFC, diamond, the other stable crystalline form of carbon, has been largely overlooked by the fusion community. The recent progress in the synthesis of artificial diamond by chemical vapour deposition (CVD) has boosted the interest and use of diamond in a large range of applications such as aerospace, biomedical engineering, electrochemistry, tribology and electronic applications. Diamond has outstanding thermal properties with a thermal conductivity of about $1800 \mathrm{~W} \mathrm{~m}^{-1} \mathrm{~K}^{-1}$, which is about five times that of copper, and a very low thermal expansion 
coefficient $\left(\sim 1 \times 10^{-6} \mathrm{~K}^{-1}\right)$. In contrast to metals where heat is conducted by electrons, heat in diamond is transported by lattice vibration. In addition, it exhibits good resistance to radiation and neutron bombardment, which makes it a good candidate for neutron detectors in ITER [4]. Moreover, due to its low reactivity with hydrogen the chemical erosion rate of diamond by thermal hydrogen atoms is about two orders of magnitude lower than that of graphite [5], although the physical sputtering yields of diamond and graphite are similar for $1 \mathrm{keV}$ hydrogen particles. Such properties may make diamond an attractive PFM for fusion and calls for a proper evaluation of its behaviour under exposure to fusion-relevant plasma conditions. In this paper, we report the results from exposure of CVD diamond coatings to deuterium plasmas in both linear plasma devices and tokamaks with a focus on the degradation mechanism of the diamond structure under plasma exposure.

\section{Experimental}

Polycrystalline diamond layers deposited on molybdenum substrates by a commercial hot-filament CVD reactor (Model 650 from $\mathrm{sp}^{3}$ Diamond Technology) were used. Details of the deposition system and the CVD process can be found elsewhere [6, 7]. Varying the deposition parameters (gas mixture composition, pressure), two types of diamond coatings were produced. The first type, named microcrystalline diamond (MCD), is composed of randomly oriented diamond grains with size between 0.5 and $2 \mu \mathrm{m}$. The second type, named nanocrystalline diamond (NCD), exhibits a typical cauliflower structure with grain size smaller than $50 \mathrm{~nm}[8]$.

The chemical erosion of diamond coatings was evaluated by a set of experiments in the Pilot-PSI [9] linear plasma device. Diamond-coated molybdenum substrates $(10 \mu \mathrm{m}$ thickness, MCD and NCD) and graphite samples were exposed to similar plasma conditions in order to compare their chemical erosion rates under exposure to high density $(3-8 \times$ $\left.10^{19} \mathrm{~m}^{-3}\right)$, low temperature $(0.3-2 \mathrm{eV})$ plasmas. The samples diameter and thickness were 25 and $2 \mathrm{~mm}$, respectively, while the diameter of the plasma column was about $13 \mathrm{~mm}$. The $\mathrm{C}-\mathrm{H}$ band emission was monitored by optical emission spectroscopy, and used to evaluate the chemical erosion. Details about the spectroscopic measurements and the data processing can be found in [10]. The plasma parameters were measured just in front of the target by a Thomson scattering system. The power arriving at the target was determined by calorimetry, the temperature rise of the cooling water circulating in the sample holder being measured by thermocouples. The target temperature, as measured by a pyrometer, was in the range $800-1000{ }^{\circ} \mathrm{C}$ in the case of graphite. No measurement was possible in the case of diamond targets, either because the temperature was below the detection limit $\left(500^{\circ} \mathrm{C}\right)$ or because of the unknown emissivity of diamond.

The sputtering yields of diamond and graphite under deuterium neutral bombardment were compared in PISCES-B. Both pure NCD coatings and boron-doped samples $(0.1 \% \mathrm{~B})$ were used. Graphite and diamond samples were installed side by side on the witness plate manipulator

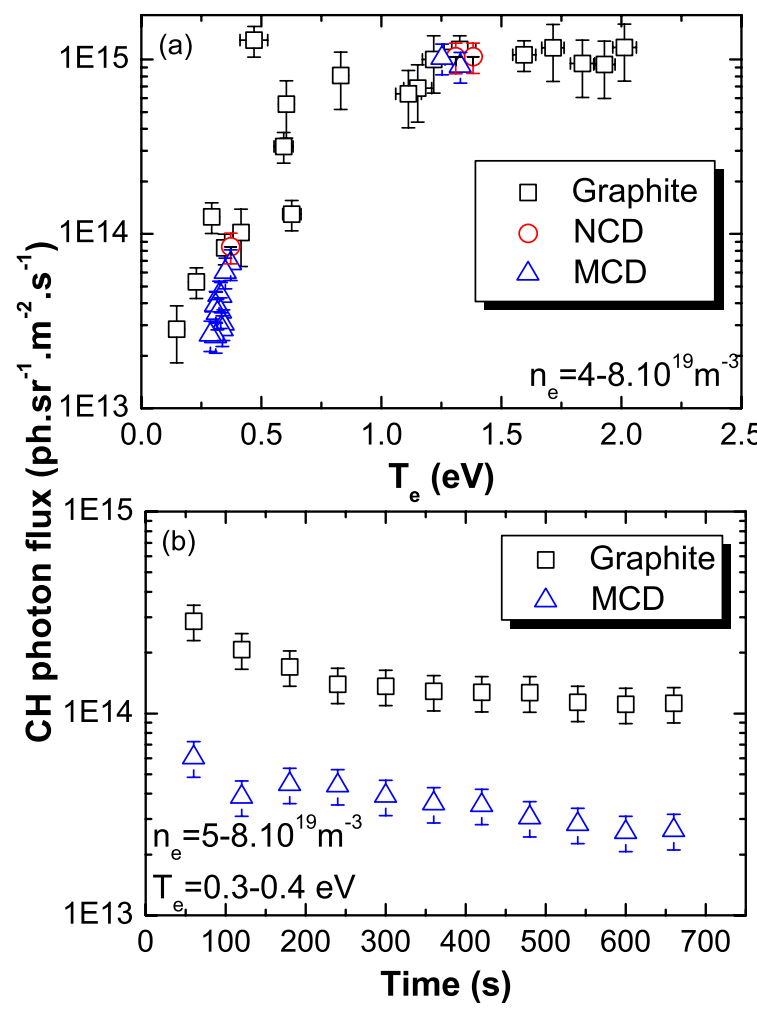

Figure 1. Evolution of the $\mathrm{CH}$ photon flux measured by optical emission spectroscopy for graphite and diamond target exposed under different plasma conditions in Pilot-PSI.

which is shielded from cross-field plasma transport and is in line of sight to the target, the experimental arrangement has been described in detail in [11]. A tungsten target was exposed to a high-flux deuterium plasma $\left(\sim 2-4 \times 10^{22}\right.$ ions $\left.\mathrm{m}^{-2} \mathrm{~s}^{-1}\right)$, and was negatively biased to control the energy of the ions impacting on the target and the energy of the reflected particles from the target. Erosion of the samples was determined by weight loss measurements. Morphology of the samples was studied by scanning electron microscopy (SEM).

Diamond-coated silicon samples were exposed in DIII-D under different plasma conditions, using the DIMES divertor probe. The first sample was exposed during detached L-mode discharges for seven shots and an equivalent of 21 plasma seconds. The sample was located near the outer strike point $\left(T_{\mathrm{e}} \sim 5 \mathrm{eV}, n_{\mathrm{e}} \sim 1 \times 10^{13} \mathrm{~cm}^{-3}\right)$. The second sample was exposed during 10 attached H-mode discharges (34 plasma seconds) with edge localized modes (ELMs) and was located $1 \mathrm{~cm}$ inboard of the outer strike point $\left(T_{\mathrm{e}} \sim 30 \mathrm{eV}, n_{\mathrm{e}} \sim 2 \times\right.$ $10^{13} \mathrm{~cm}^{-3}$ ). Sample erosion was evaluated by nuclear reaction analysis (NRA), secondary ion mass spectrometry (SIMS) and profilometry.

\section{Results}

\subsection{Erosion of diamond}

The chemical erosion of graphite and diamond was compared in Pilot-PSI by measuring spectroscopically the intensity of the $\mathrm{CH}$ emission band. Figure 1(a) shows the evolution of the $\mathrm{CH}$ emission from graphite and diamond coatings (both MCD and NCD) as a function of the electron temperature in front of the target. The samples were at the floating potential, 
Table 1. Comparison of the weight loss measurements for ATJ graphite and diamond samples, pure NCD, and boron-doped MCD $(0.1 \% \mathrm{~B})$, after exposure to deuterium neutrals in PISCES-B.

\begin{tabular}{lcc}
\hline Sample & $E_{\mathrm{n}}(\mathrm{eV})$ & Mass loss $(\mathrm{mg})$ \\
\hline Graphite & 25 & $290 \pm 50$ \\
NCD & 25 & $270 \pm 0$ \\
Doped MCD $(0.1 \% \mathrm{~B})$ & 25 & $130 \pm 50$ \\
\hline
\end{tabular}

which was measured during the plasma exposure. However, since diamond is an insulator, no such measurement was possible for diamond. The plasma conditions being similar for the three samples, it is assumed that the floating potential and the energy of the incoming ions are identical for the three cases. For all samples, it is found that the $\mathrm{CH}$ emission increases with the electron temperature and saturates for $T_{\mathrm{e}} \geqslant 1 \mathrm{eV}$. A similar trend has been observed for various grades of carbon materials (CFC, graphite) and is further discussed in [10]. It should be noted that the inverse photon efficiency for $\mathrm{CH}$ has been found to be constant in the range of electron temperatures investigated here [12]. The NCD and MCD coatings show a reduced chemical erosion compared to graphite by a factor of about 2, at least for electron temperatures below $1 \mathrm{eV}$. $\mathrm{CH}$ emission from NCD coatings appears higher than that of MCD coatings, a similar higher erosion of NCD coatings has been observed in PISCES-B and will be discussed later. One has to keep in mind, however, the large uncertainty in the temperature of the diamond target.

In a second series of experiments, the same plasma $(60 \mathrm{~s}$ duration) was repeated 11 times and the evolution of the $\mathrm{CH}$ emission was followed as a function of the accumulated exposure time. As seen in figure 1(b), a large decrease of the $\mathrm{CH}$ emission is observed for graphite after the first four shots, with a slower continuous decrease after the fifth shot. In the case of diamond, no sharp decrease is measured at the beginning of the sequence but a monotonic decrease of the $\mathrm{CH}$ emission is also observed. After the initial sharp drop, the $\mathrm{CH}$ emission for diamond is about four times lower than that of graphite. A monotonic decrease of the chemical erosion yield with ion fluence is usually observed for doped graphite samples and is explained by progressive enrichment of the surface with doping atoms [13]. In the present case, the presence of metallic impurities $(\mathrm{W}, \mathrm{Cu})$, likely originating from atoms sputtered in the plasma source, was evidenced by $\mathrm{X}$-ray photoelectron spectroscopy (XPS) measurements after exposure and might explain the observed trend.

The uncertainty in the surface temperature of the diamond samples during exposure adds an uncertainty in the previous observations since the chemical erosion yield strongly depends on the temperature [14]. The graphite samples temperature (1000-1200 K) was well above the peak temperature for chemical erosion. If the temperature of the diamond samples was close to the peak temperature, the difference between graphite and diamond would be much larger than that reported here, which would be consistent with recent measurements of the chemical erosion yield of diamond coated graphite by low temperature plasmas $(\sim 1 \mathrm{eV})$ [15].

Table 1 shows the measured erosion of ATJ graphite, NCD and boron-doped MCD $(0.1 \%$ B) after exposure to deuterium neutrals reflected from a tungsten target. Within the accuracy of the measurements, nano-crystalline coatings did not behave differently from graphite. On the other hand, doped diamond showed a factor 2.2 lower erosion than graphite. The higher erosion of the NCD is attributed to the relatively high content of $\mathrm{sp}^{2}$ carbon usually found in that type of coating [16], mainly at the grain boundaries. The influence of the doping element could not be distinguished here and will have to be assessed in future experiments. Doped samples are, however, more interesting for fusion because of their electrical conductivity. Indeed, strong arcing was observed after the exposure of pure diamond coatings in Mega-Amp Spherical Tokamak (MAST) [6].

Finally, no measurable erosion was observed for NCD coatings exposed under detached plasma conditions in DIII-D, while under attached ELMing conditions, around $300 \mathrm{~nm}$ of the layer was eroded in 34 plasma seconds, yielding an erosion rate of about $10 \mathrm{~nm} \mathrm{~s}^{-1}$. In the latter case, the much higher plasma temperature naturally gives rise to a much higher erosion rate than under detached plasma conditions where $T_{\mathrm{e}}$ is very low. It is not possible at this stage to assess the effect of ELMs on the global erosion.

\subsection{Modification of the diamond structure under particle bombardment}

Figure 2(a) shows the typical morphology of a $10 \mu \mathrm{m}$ thick micro-crystalline coating, exhibiting randomly oriented grains with size around $0.5-1 \mu \mathrm{m}$. After exposure to a deuterium plasma in Pilot-PSI, with a total fluence of $\sim 7 \times 10^{24}$ ions $\mathrm{m}^{-2}$, the morphology of the surface is strongly modified (figure 2(b)). The grain boundaries appear much less distinctly and the nicely defined grain structure shown in figure 2(a) has almost disappeared. In addition, some white spots are visible on the surface which, according to EDX analyses, appear to be metallic nano-particles made of tungsten and copper, amongst other elements. The likely source of these metallic impurities is the sputtering of the plasma source of Pilot-PSI and the subsequent transport of these impurities to the target by the plasma flow. In the case of the NCD sample (not shown here), the cauliflower structure of the coating is still observed [16] but a strong etching is observed at the grain boundaries where $\mathrm{sp}^{2}$ carbon is usually observed. On the other hand, no change of the morphology is observed for the boron-doped layer exposed in PISCES-B for which well-defined grains are still observed and have not been modified by particle bombardment.

Both Raman spectroscopy and XPS were used to evaluate the change in the samples crystallographic structure induced by the plasma exposure. Figure 3(a) shows the $\mathrm{C} 1$ s core level spectrum of a MCD sample after exposure in Pilot-PSI, with $T_{\mathrm{e}}=0.5 \mathrm{eV}$, which is actually identical to the spectrum measured before exposure. Deconvolution of the spectrum shows the prominence of the peak corresponding to $\mathrm{sp}^{3}$ carbon (i.e. diamond). The presence of other chemical states of carbon is also found, but no clear deconvolution can be done, because of the low intensity of these different peaks associated with the finite resolution of the spectrometer. Since the sample had been exposed to air, and given that those additional peaks are shifted towards higher binding energies, this can be attributed to different carbon-oxygen 


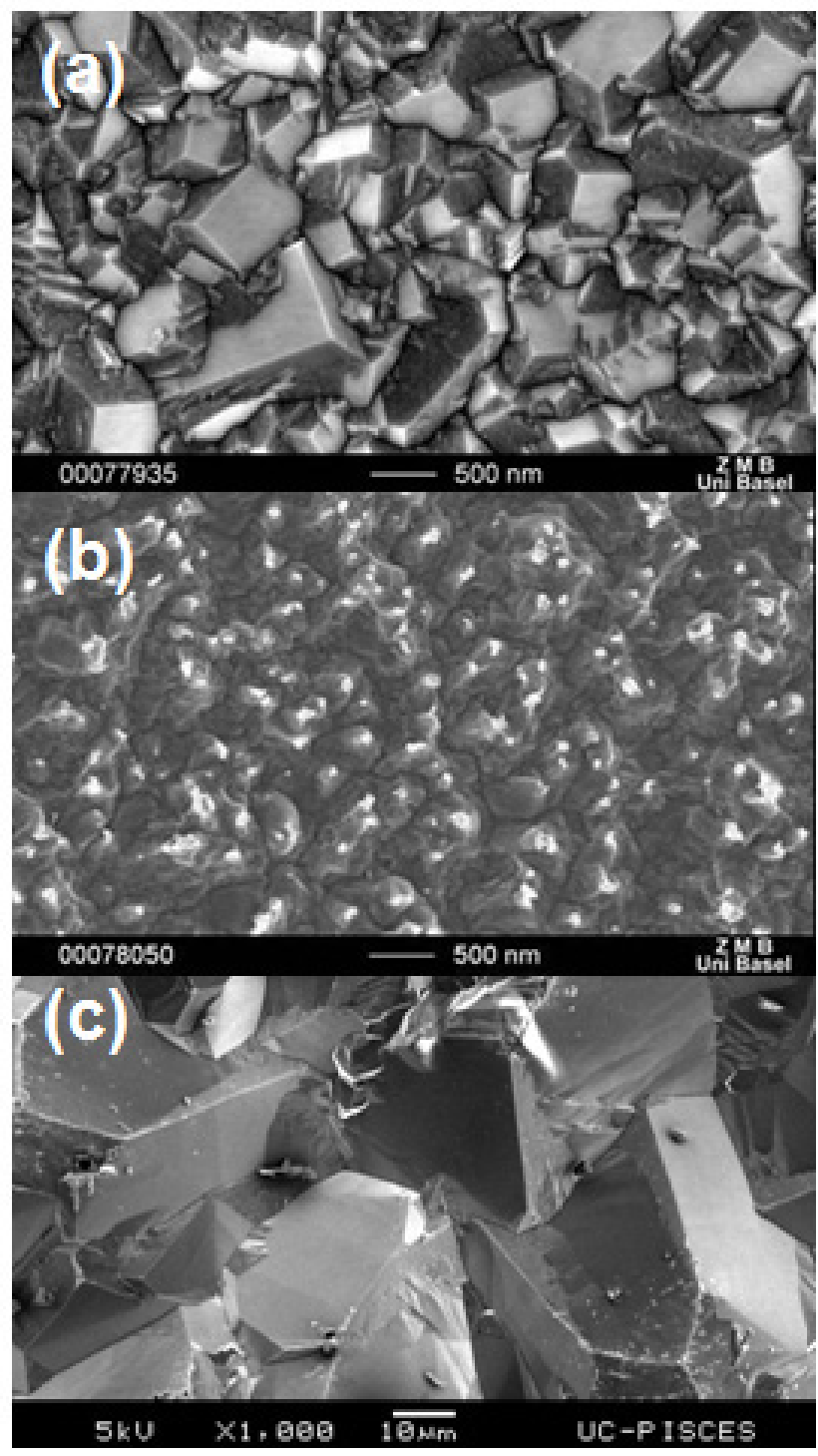

Figure 2. SEM pictures of pure micro-crystalline diamond samples (a) as-deposited, (b) after exposure in Pilot-PSI and (c) of a boron-doped micro-crystalline sample after exposure in PISCES.

bonds. For higher electron temperatures (figure 3(b)), a peak corresponding to $\mathrm{sp}^{2}$ carbon is found, shifted towards lower energy compared to the diamond peak. Similar observations were made for samples exposed in DIII-D, i.e. no modification of the $\mathrm{C} 1 \mathrm{~s}$ core level spectrum was measured for the sample exposed under detached conditions, while a partial amorphization (appearance of $\mathrm{sp}^{2}$ carbon) of the surface occurred for the samples exposed under ELMy H-mode conditions. On the other hand, as shown in figure 4, Raman spectroscopy measurements of the MCD samples exposed in Pilot did not evidence any significant modifications of the bulk of the samples. Both the diamond peak (at $1333 \mathrm{~cm}^{-1}$ ) and the G-band at $1570 \mathrm{~cm}^{-1}$ (corresponding to graphitic carbon) are identical for the unexposed and exposed samples. However, for the sample shown in figure 3(b), a shift of the diamond peak from 1333 to $1336 \mathrm{~cm}^{-1}$ is observed, which is assumed to be due to strains induced in the film by the plasma exposure. As expected, amorphization of the diamond structure only occurs within the penetration depth of the incoming particles and the bulk remains unaffected under the

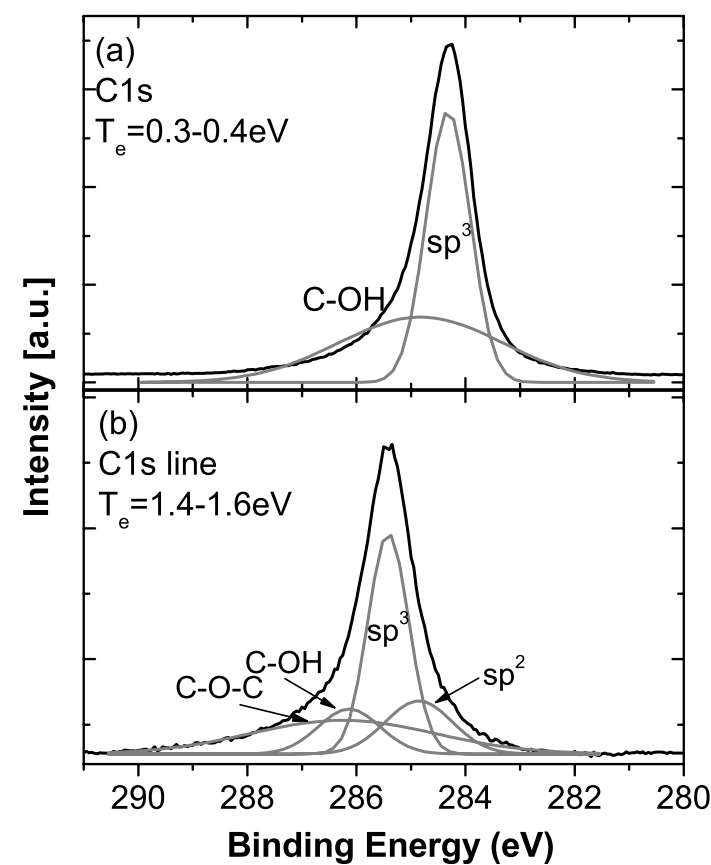

Figure 3. XPS measurements of the $\mathrm{C} 1 \mathrm{~s}$ core level spectra of a MCD coatings after exposure in Pilot-PSI with (a) $T_{\mathrm{e}} \sim 0.5 \mathrm{eV}$ and (b) $T_{\mathrm{e}} \sim 1.5 \mathrm{eV}$. Note that the shift in binding energy between those two samples is due to the insulating properties of diamond and subsequent charging of the samples.

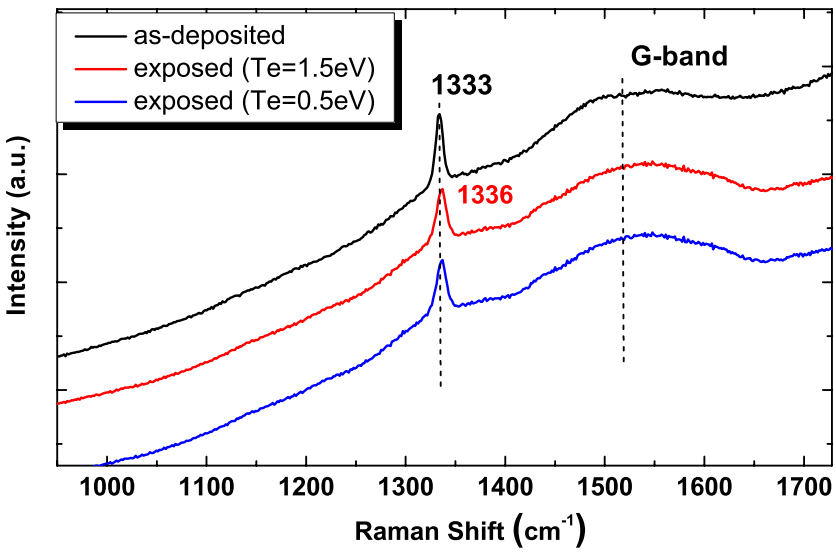

Figure 4. Raman spectra (laser excitation wavelength $514 \mathrm{~nm}$ ) of a MCD sample before and after exposure in Pilot-PSI under different conditions.

investigated conditions. The amorphization of this interaction zone will lead to an increase of the chemical erosion rate of diamond. Indeed, lattice damage created by transfer of kinetic energy from the incoming particles to the lattice atoms will provide open bonds for hydrogen attachment [17]. Earlier experiments have shown, however, that the chemical sputtering yield of diamond by hydrogen ions with energies in the range $0.2-0.8 \mathrm{keV}$, remains lower than that of graphite [18] by about $50 \%$. The lower the incident particle energy the larger the difference between chemical erosion of graphite and diamond.

\section{Conclusions}

First experimental investigations of diamond coatings under fusion-relevant conditions have shown that diamond has some 
properties which could make it a suitable PFM for a nuclear fusion reactor. Within the range of this study, the sputtering yield of diamond appears to be about two times lower than that of graphite. Under detached plasma conditions, no measurable erosion was found for a diamond coating exposed in the DIII-D divertor, whereas strong erosion was found after exposure to an attached ELMy H-mode plasma. In addition, some particle-bombardment-induced amorphization of the diamond coatings has been observed but is limited to the penetration depth of the incident particles leaving the bulk material intact. It is therefore expected that the thermal properties of the bulk, which overpass those of other materials, will be maintained. The first results being rather encouraging, dedicated experiments are planned in TEXTOR and in JUDITH to study the influence of the boron content in a doped layer on the sputtering yield and to assess the performance of diamond under repetitive transient heat loads.

\section{Acknowledgments}

This work was funded jointly by the UK EPSRC and by the European Communities under the contract of Association between EURATOM and UKAEA. The views and opinions expressed herein do not necessarily reflect those of the European Commission.

Published under licence from EURATOM.

\section{References}

[1] Baluc N et al 2006 Plasma Phys. Control. Fusion 48 B165

[2] Stoneham A M, Matthews J R and Ford I J 2004 J. Phys.: Condens. Matter. 16 S2597

[3] Mirnov S 2009 J. Nucl. Mater. 390-391 876

[4] Pillon M et al 2008 J. Appl. Phys. 104054513

[5] Donelly C M et al 1997 Diam. Relat. Mater. 6787

[6] Porro S et al 2009 Diam. Relat. Mater. 18740

[7] Herlinger J 2006 Thin Solid Films 59165

[8] Williams O A et al 2008 Diam. Relat. Mater. 171080

[9] van Rooij G J et al 2007 Appl. Phys. Lett. 90121501

[10] Westerhout J et al 2009 Phys. Scr. T138 014017

[11] De Temmerman G et al 2008 Nucl. Fusion 48075008

[12] Westerhout J et al 2009 Appl. Phys. Lett. 95151501

[13] Balden M et al 2003 J. Nucl. Mater. 313-316 348

[14] Roth J et al 1999 J. Nucl. Mater. 266-269 51

[15] Takeguchi Y et al 2009 Phys. Scr. T138 014056

[16] Porro S et al 2009 Phys. Stat. Sol. a 2062028

[17] Roth J and Garcia-Rosales C 1996 Nucl. Fusion 36647

[18] Yamada R 1987 J. Vac. Sci. Technol. 52222 